\title{
The practical use of acetylsalicylic acid in the era of the ASPRE trial. Update and literature review
}

\author{
Przemyslaw Kosinski ${ }^{1}$, Urszula Sarzynska-Nowacka ${ }^{1}$, Magdalena Fiolna ${ }^{2}$, Miroslaw Wielgos ${ }^{1}$ \\ ${ }^{1} 7{ }^{\text {st }}$ Department of Obstetrics and Gynecology, Medical University of Warsaw, Poland \\ ${ }^{2}$ Fetal Medicine Unit, Medway Maritime Hospital, Gillingham, Kent, United Kingdom
}

\begin{abstract}
It is now well established that acetylsalicylic acid — one of the most widely prescribed drugs today — has brought a new era in maternal-fetal medicine. The History of medicine mentions several antecedents. Extracts made from willow contained in clay tablets are reported in both ancient Sumer and Egypt. In 400 BC, Hippocrates referred to the use of salicylic tea to reduce fevers. In the 1950s, acetylsalicylic acid entered the Guinness Book of Records as the highest selling painkiller. There is little doubt that acetylsalicylic acid — one of the first drugs to enter common usage — remains one of the most researched drugs in the world.
\end{abstract}

Ginekologia Polska 2018; 89, 2: 107-111

\section{INTRODUCTION}

Hypertension complicates approximately $10 \%$ of all pregnancies, including 2-3\% of preeclamptic gestations [1]. Preeclampsia (PE) remains a major concern for all obstetricians around the world. It is a systemic vascular disorder, characterized by the onset of hypertension in a previously normotensive patient, and is accompanied by proteinuria or multiple organ failure, developing after 20 weeks of gestation.

Contributing causes of PE development include: abnormal transformation of the spiral arteries during placentation, impaired placental perfusion, hypoxia, and ischemia (which results in an excess of angiogenic factors, i.e. soluble fms-like tyrosine kinase [sFlt-1] and soluble endoglin [sEng], which lead to increased vascular permeability and blood vessel contraction, as well as the activation of a coagulation cascade). Analysis of the blood flow in maternal arteries is a good detector of pregnant women at a high-risk of PE. Blood flow resistance in maternal arteries decreases in the course of an uncomplicated pregnancy and significantly increases in patients with a high risk of PE [2]. As a result, hypertension, proteinuria, and other clinical manifestations have been observed [3]. Acetylsalicylic acid appeared at the end of the 19th century as a synthetic analgesic agent with improved gastric tolerability vs. naturally occurring salicylates. It was marketed as aspirin in 1899 and turned out to be a good antiplatelet agent. Not so long ago, it was also described as a safe and effective drug to reduce the rate of $P E$ in high-risk pregnant women $[3,4]$.

\section{ACETYLSALICYLIC ACID IN FETAL MEDICINE}

Following reports on aspirin as an effective anti-inflammatory and anti-platelet agent, observational studies on pregnant women were performed. Crandon and Isherwood (1979) showed that a regular intake of aspirin was linked to a lower incidence of PE. Beaufils et al. (1985) suggested that antiplatelet therapy - given early in pregnancy to high-risk patients - may protect against PE and fetal growth restriction [5]. PE is still a common cause of both maternal and fetal complications. The only effective method of treatment - delivery - results in prematurity and the subsequent complications. Therefore, preventive measures are of the utmost importance to lower the risk of PE. The pathogenesis is complex and all interventions so far have not generated 
the expected results in the general population of pregnant women. Low doses (60-150 mg) of acetylsalicylic acid in high-risk patients seem to be the only effective method. It has been observed that PE is associated with elevated levels of platelet thromboxane, which led to a number of studies on the role of acetylsalicylic acid in disease prevention among high-risk women. In contrast to high doses, low doses (60-150 mg) of acetylsalicylic acid reduce thromboxane production, while preserving the synthesis of prostacyclin in the vascular walls [6]. In 1982, the inhibition of prostacyclin formation was alleged to be a facilitator of both thrombosis and obstetrical problems in patients with lupus anticoagulant. This opened a new chapter in maternal-fetal medicine - a prophylaxis of conditions named thrombophilia. Aspirin in low doses is the single most cost-effective medicine for the prevention of the secondary events of thrombosis [7].

\section{ASPIRIN - A "NEW" MECHANISM OF ACTION}

Since acetylsalicylic acid is long established as an analgesic, antipyretic, and antiplatelet drug, alternative modes of action have received insufficient recognition, up until now. Interestingly, aspirin induces the production of pro-resolving lipid-derived mediators - similar to endogenous mediators. These biochemical agents are built from arachidonic acid (AA), omega-3 polyunsaturated fatty acids (PUFAs), and aspirin-triggered lipoxins (ATLs), binding to a dedicated receptor (ALXR) [7]. ATLs act as antioxidant and immunomodulators, promoting inflammation resolution, and inhibiting blood vessel proliferation [7, 8]. Increases of both ALXR expression in the human decidua, and of serum lipoxin A4 level during the first trimester of pregnancy, may be a starting point in the understanding of aspirin effectiveness in PE or placental pathologies [9]. It is hypothesized that early (before $34+0$ weeks, around $20 \%$ of cases) and late (after $34+0$ weeks, around $80 \%$ of cases) onset PE have different etiologies, but a common place of origin - the placenta [10]. Considering the high prevalence of $\mathrm{PE}$, and its serious complications both for mothers and newborns, prediction and prophylaxis represent twin-challenges in modern obstetrics.

\section{A "LOW-DOSE" ASPIRIN - HOW MUCH IS ENOUGH?}

International associations recommend $60-80 \mathrm{mg}$ of aspirin for women at high risk of developing PE. In 2014, the United States Preventive Services Task Force (USPTF) published a meta-analysis employing doses of between 49-150 mg, and there was no evidence of a dose-response relationship [11]. A dosage of at least $75 \mathrm{mg}$ per day had a greater benefit in reducing the risk of preeclampsia than a dosage of less than $75 \mathrm{mg}$ per day (risk ratio [RR], 0.58 [95\% confidence interval (CI), 0.36-0.95]; and
$\mathrm{RR}, 0.85$ [95\% Cl, 0.68-1.05] respectively). An $81 \mathrm{mg}$ dose emerged because of its availability in the United States and being within a low-dose range (75-150 mg). Guidance issued to patients - for the use of different low-doses of aspirin to prevent PE - is summarized in Table 1 (modified from Jackson\&Gregg, 2017) [12]. Aspirin resistance research has also increased notably in recent years with pregnant women $[13,14]$. Recently, reports supporting doses reaching the upper values of the "low-dose" range have emerged, and a meta-analysis of forty-five RCTs - including a total of 20,909 pregnant women showing a dose-dependent benefit to therapy — was published [15]. Higher dosages initiated $<16$ weeks of gestation and were associated with a greater reduction of all three outcomes $(\mathrm{PE}$, severe $\mathrm{PE}$, and fatal growth restriction). Since meta-analyses are the most powerful tool in implementing recommendations, the authors take up the position of prescribing $150 \mathrm{mg}$ of aspirin per day.

\section{CIRCADIAN TIMING OF ASPIRIN}

Chronology in aspirin administration was once an issue. A double-blind placebo-controlled trial on pregnant women at high risk of $\mathrm{PE}$, and a prospective trial on patients with untreated mild hypertension, both concluded that a low-dose of acetylsalicylic acid lowers blood pressure when administered at bedtime and not on waking $[16,17]$. In another trial, the authors conducted a prospective, randomized, double-blind, placebo-controlled, chronotherapy trial on 350 high-risk pregnant women $13.5 \pm 1.4$ weeks of gestation at the time of recruitment [18], finding that a low-dose aspirin ingested at bedtime, but not on waking, significantly regulates ambulatory blood pressure and reduces the incidence of $\mathrm{PE}$, gestational hypertension, preterm delivery, and IUGR. Interestingly, the described effect was expressed to a larger extent in women, patients with elevated fasting glucose, and a high glomerular filtration rate [19].

\section{VALUABLE NOT ONLY IN PREECLAMPSIA}

Fetal growth restriction (FGR) and PE are thought to be different syndromes of the same disorder: placental dysfunction. Ness and Sibai hypothesized that endothelial dysfunction precedes abnormal placentation, and that both are cornerstones of PE and FGR pregnancies. They suggest that the particular clinical manifestation is determined by certain metabolic disturbances [20].

A low aspirin dosage (50-150 mg/day) - started 16 weeks before gestation in high-risk woman — was associated with a significant reduction in perinatal death and FGR, with a mean birth weight increment of $209 \mathrm{~g}$ (95\% Cl: 100-319) [21]. A meta-analysis of 17 randomized trials showed a $50 \%$ reduction of FGR risk (RR: $0.47 ; 95 \% \mathrm{Cl}$ : $0.36-0.62, \mathrm{P}<.01)$ using $60-150 \mathrm{mg}$ of aspirin/day [22]. 
Table 1. Selected organization guidance on patients who are appropriate candidates for the use of different low-doses of aspirin to prevent preeclampsia (modified from Jackson\&Gregg 2017 [12])

\begin{tabular}{|c|c|c|c|c|}
\hline & USPTF 60-150 mg [11] & ACOG 60-80 mg [28] & NICE 75 mg [29] & WHO $75 \mathrm{mg}$ [30] \\
\hline \multicolumn{5}{|c|}{ High risk $>1$} \\
\hline History of PE & $x$ & $x$ & $\mathrm{x}$ & $\mathrm{x}$ \\
\hline Multifetal gestation* & $x$ & $x$ & - & $x$ \\
\hline Chronic hypertension & $\mathrm{x}$ & $\mathrm{x}$ & $\mathrm{x}$ & $\mathrm{x}$ \\
\hline Diabetes type 1 or 2 & $x$ & $x$ & $x$ & $x$ \\
\hline Renal disease & $x$ & $x$ & $x$ & $x$ \\
\hline Autoimmune disease & $x$ & $x$ & $x$ & $x$ \\
\hline \multicolumn{5}{|c|}{ Moderate risk $>1 * *$} \\
\hline Nulliparous & $\mathrm{x}$ & - & $\mathrm{x}$ & - \\
\hline Age $>40$ years & - & - & $x$ & - \\
\hline$>10$ years IPI & $x$ & - & $x$ & - \\
\hline First degree relative & $x$ & - & $x$ & - \\
\hline $\mathrm{BMI}>35 \mathrm{~kg} / \mathrm{m} 2$ & - & - & $x$ & - \\
\hline Multifetal gestation & - & - & $x$ & - \\
\hline Low SES & $x$ & - & - & - \\
\hline Age $>35$ years & $x$ & - & - & - \\
\hline $\mathrm{BMl}>30 \mathrm{~kg} / \mathrm{m} 2$ & $x$ & - & - & - \\
\hline Patient born LBW & $x$ & - & - & - \\
\hline Prior APO & $\mathrm{x}$ & - & - & - \\
\hline
\end{tabular}

ACOG — American College of Obstetricians and Gynecologists; APO — adverse pregnancy outcome (not specifically defined); BMI — body mass index;

IPI - interpregnancy interval; LBW — low birth weight; NICE — National Institute for Health and Care Excellence (United Kingdom); PE — physical education;

SES - socioeconomic status (not specifically defined); USPTF — U.S. Preventive Services Task Force; WHO — World Health Organization

* Multifetal gestation is not a high-risk factor but is a moderate-risk factor in the NICE guidance

** Moderate-risk factors are those with less than $8 \%$ risk. NICE proposes 2 or more for aspirin prophylaxis

\section{THE NEW ERA OF PE PROPHYLAXIS - THE ASPRE STUDY}

Although many studies have demonstrated the benefits of aspirin administration during pregnancy, the first randomized controlled trials failed to prove any remarkable advantages of such prophylaxis [23]. For this reason, a large, multicentre randomized, placebo-controlled European Trial (ASPRE) was conducted by Professor Kypros Nicolaides of King's College Hospital in London. The results have recently been published in The New England Journal of Medicine [24]. A group of 1776 women in singleton pregnancies, at a high risk of $\mathrm{PE}$, were randomly assigned to receive either aspirin at a dose of $150 \mathrm{mg}$ or placebo, starting from 11 to 14 weeks of gestation until 36 weeks of gestation. The study was designed to answer the question regarding aspirin influence on $\mathrm{PE}$, which arose from ambiguous meta-analyses. Firstly, the authors took an alternative approach to their screening method, the effectiveness of which was validated in a study involving approximately 60,000 women. The combined multimarker screening is a combination of a priori risk together with medical information acquired at 11-13 weeks of gestation, employing Bayes'theorem. This detects $76 \%$ of preterm cases and $38 \%$ of term PE [25]. The trial was distinguished from other aspirin-based studies by the high accuracy across the range of high-risk patients. The variables calculated in the algorithm were: age, height, weight, racial origin, chronic hypertension, systemic lupus erythematosus or antiphospholipid syndrome, method of conception, parity in regard to the history of PE and intergestational interval, family history of $\mathrm{PE}$, diabetes mellitus type 1 or 2 , cigarette smoking, mean arterial pressure, uterine artery pulsatility index, pregnancy associated plasma protein-A (PAPP-A), and 
placental growth factor (PIGF). Moreover, the authors looked for preterm rather than total PE. A threshold for "high-risk" pregnancy was established at $>$ 1:100 and delivery with PE before 37 weeks of gestation was measured as a primary outcome. The participants were randomly assigned to aspirin or placebo groups, in a 1:1 ratio. PE before 37 weeks' gestation was diagnosed in 13 of 798 (1.6\%) patients in the aspirin group and 35 of 822 (4.3\%) in the placebo group (adjusted odds ratio in the aspirin group $-0.38 ; 95 \% \mathrm{Cl} ; 0.2$ to 0.74 ; $\mathrm{P}=0.004)$. The trial was not sufficiently powered for secondary outcomes; nevertheless, the incidence of pregnancy, or fetal or neonatal complications, were similar in both groups. The authors chose aspirin at a dose of $150 \mathrm{mg}$, on the basis of a previously justified dose-dependent therapeutic benefit. In conclusion, $150 \mathrm{mg}$ of aspirin given at bedtime at 11 to 14 weeks of gestation, until 36 weeks, significantly lowered the incidence of developing preterm $\mathrm{PE}$, compared to placebo, providing a basis to unify recommendations on aspirin commencement. It is worth mentioning that, in this study, aspirin did not lower the risk of term PE.

Based on the conducted studies and histopathological observations, it seems justified to initiate treatment before 16 weeks of gestation as placental implantation and transformation of the maternal spiral arteries are completed between 16 and 20 weeks of gestation. Thus, it seems that the beneficial effect of acetylsalicylic acid is the consequence of improved spiral artery transfer [21]. This hypothesis is based on two factors: i) low doses of acetylsalicylic acid are most effective where early-pregnancy PE prevention is concerned, which is mostly connected with impaired placental implantation [26], and ii) abnormal blood flow in maternal arteries - already observed at 12 weeks of gestation, in women who will later develop PE - significantly improves between the first and the second trimester in patients receiving acetylsalicylic acid.

\section{SUMMARY}

Preeclampsia remains one of the most serious complications in pregnancy. Hopefully, in the near future, patients attending their first visit (between 12-14 weeks of gestation) will be offered screening tests for both PE and genetic abnormalities. The current state of knowledge suggests that only pre-selected high-risk patients should receive preventive low-doses of acetylsalicylic acid. Resistance to acetylsalicylic acid remains an area of debate. As many as 30\% of pregnant women are suspected to be aspirin-resistant. For this reason, in centres such as King's College Hospital, a dosage of $150 \mathrm{mg}$ of acetylsalicylic acid at bedtime is used, which is a compromise between the dose being effectively therapeutic and the risk of possible adverse effects. The belief that $\mathrm{PE}$ is a condition limited to pregnancy - with the risk of termination after placenta removal — is no longer held true. Women with a history of PE have an increased risk of ischemic heart disease, stroke, cerebrovascular disease, peripheral arterial disease, cardiovascular mortality and death from stroke, chronic hypertension, end-stage renal disease, and metabolic disorders. Aspirin is a well-known preventive agent diminishing the risk of many of these conditions. Future research could be focussed on post-pregnancy aspirin use. At the same time, it is important to emphasize that screening for PE and pharmacological prophylaxis using acetylsalicylic acid requires further, multi-centre randomized trials in order to implement both screening tests and preventive measures into daily clinical practice.

\section{REFERENCES}

1. Benigni A, Gregorini G, Frusca T, et al. Effect of low-dose aspirin on fetal and maternal generation of thromboxane by platelets in women at risk for pregnancy-induced hypertension. N Engl J Med. 1989; 321(6): 357-362, doi: 10.1056/NEJM198908103210604, indexed in Pubmed: 2664523.

2. Kosiński P, Samaha RB, Bomba-Opoń DA, et al. Reference values for placental growth factor (PIGF) concentration and uterine artery doppler pulsatility index (PI) at 11-13(+6) weeks of gestation in the Polish population. Ginekol Pol. 2014; 85(7): 488-493, doi: 10.17772/gp/1758, indexed in Pubmed: 25118498.

3. Bujold $E$, Roberge $S$, Lacasse $Y$, et al. Prevention of preeclampsia and intrauterine growth restriction with aspirin started in early pregnancy: a meta-analysis. Obstet Gynecol. 2010; $116(2$ Pt 1): 402-414, doi: 10.1097/AOG.0b013e3181e9322a, indexed in Pubmed: 20664402.

4. Patrono C, Rocca B. Aspirin, 110 years later. J Thromb Haemost. 2009; 7 Suppl 1: 258-261, doi: 10.1111/j.1538-7836.2009.03391.x, indexed in Pubmed: 19630812

5. Beaufils M, Uzan S, Donsimoni R, et al. Prevention of pre-eclampsia by early antiplatelet therapy. Lancet. 1985; 1(8433): 840-842, doi: 10.1016/s0140-6736(85)92207-x, indexed in Pubmed: 2858710.

6. Dekker GA, Sibai BM. Low-dose aspirin in the prevention of preeclampsia and fetal growth retardation: rationale, mechanisms, and clinical trials. Am J Obstet Gynecol. 1993; 168(1 Pt 1): 214-227, indexed in Pubmed: 8420330.

7. Cadavid AP. Aspirin: The Mechanism of Action Revisited in the Context of Pregnancy Complications. Front Immunol. 2017; 8: 261, doi: 10.3389/fimmu.2017.00261, indexed in Pubmed: 28360907.

8. Parkinson JF. Lipoxin and synthetic lipoxin analogs: an overview of anti-inflammatory functions and new concepts in immunomodulation. Inflamm Allergy Drug Targets. 2006; 5(2): 91-106, doi: 10.2174/187152806776383125, indexed in Pubmed: 16613568.

9. Macdonald LJ, Boddy SC, Denison FC, et al. A role for lipoxin $A_{4}$ as an anti-inflammatory mediator in the human endometrium. Reproduction. 2011; 142(2): 345-352, doi: 10.1530/REP-11-0021, indexed in Pubmed: 21555360.

10. Huppertz B. Placental origins of preeclampsia: challenging the current hypothesis. Hypertension. 2008; 51(4): 970-975, doi: 10.1161/HYPERTENSIONAHA.107.107607, indexed in Pubmed: 18259009.

11. Henderson JT, Whitlock EP, O'Connor E, et al. Low-dose aspirin for prevention of morbidity and mortality from preeclampsia: a systematic evidence review for the U.S. Preventive Services Task Force. Ann Intern Med. 2014; 160(10): 695-703, doi: 10.7326/M13-2844, indexed in Pubmed: 24711050.

12. Jackson JR, Gregg AR. Updates on the Recognition, Prevention and Management of Hypertension in Pregnancy. Obstet Gynecol Clin North Am. 2017; 44(2): 219-230, doi: 10.1016/j.ogc.2017.02.007, indexed in Pubmed: 28499532.

13. Al-Jabi SW. Global Trends in Aspirin Resistance-Related Research from 1990 to 2015: A Bibliometric Analysis. Basic Clin Pharmacol Toxicol. 2017; 121(6): 512-519, doi: 10.1111/bcpt.12840, indexed in Pubmed: 28667787.

14. Navaratnam K, Alfirevic A, Alfirevic Z. Low dose aspirin and pregnancy: how important is aspirin resistance? BJOG. 2016; 123(9): 1481-1487, doi: 10.1111/1471-0528.13914, indexed in Pubmed: 26929162. 
15. Roberge $\mathrm{S}$, Nicolaides $\mathrm{K}$, Demers $\mathrm{S}$, et al. The role of aspirin dose on the prevention of preeclampsia and fetal growth restriction: systematic review and meta-analysis. Am J Obstet Gynecol. 2017; 216(2): 110-120. e6, doi: 10.1016/j.ajog.2016.09.076, indexed in Pubmed: 27640943.

16. Hermida RC, Ayala DE, Mojón A, et al. Ambulatory blood pressure control with bedtime aspirin administration in subjects with prehypertension. Am J Hypertens. 2009; 22(8): 896-903, doi: 10.1038/ajh.2009.83, indexed in Pubmed: 19407805.

17. Hermida RC, Ayala DE, Iglesias M. Administration time-dependent influence of aspirin on blood pressure in pregnant women. Hypertension. 2003; 41(3 Pt 2): 651-656, doi: 10.1161/01.HYP.0000047876.63997.EE, indexed in Pubmed: 12623974.

18. Ayala DE, Ucieda R, Hermida RC. Chronotherapy with low-dose aspirin for prevention of complications in pregnancy. Chronobiol Int. 2013; 30(1-2): 260-279, doi: 10.3109/07420528.2012.717455, indexed in Pubmed: 23004922.

19. Ayala DE, Hermida RC. Sex differences in the administration-time-dependent effects of low-dose aspirin on ambulatory blood pressure in hypertensive subjects. Chronobiol Int. 2010; 27(2): 345-362, doi: 10.3109/07420521003624662, indexed in Pubmed: 20370474.

20. Ness RB, Sibai BM. Shared and disparate components of the pathophysiologies of fetal growth restriction and preeclampsia. Am J Obstet Gynecol. 2006; 195(1): 40-49, doi: 10.1016/j.ajog.2005.07.049, indexed in Pubmed: 16813742
21. Roberge $\mathrm{S}$, Nicolaides $\mathrm{KH}$, Demers $\mathrm{S}$, et al. Prevention of perinatal death and adverse perinatal outcome using low-dose aspirin: a meta-analysis. Ultrasound Obstet Gynecol. 2013; 41(5): 491-499, doi: 10.1002/uog.12421, indexed in Pubmed: 23362106.

22. Roberge $\mathrm{S}$, Odibo AO, Bujold E. Aspirin for the Prevention of Preeclampsia and Intrauterine Growth Restriction. Clin Lab Med. 2016; 36(2): 319-329, doi: 10.1016/j.cll.2016.01.013, indexed in Pubmed: 27235915.

23. Caritis S, Sibai B, Hauth J, et al. Low-dose aspirin to prevent preeclampsia in women at high risk. National Institute of Child Health and Human Development Network of Maternal-Fetal Medicine Units. N Engl J Med. 1998; 338(11): 701-705, doi: 10.1056/NEJM199803123381101, indexed in Pubmed: 9494145.

24. Rolnik DL, Wright $D$, Poon LC, et al. Aspirin versus Placebo in Pregnancies at High Risk for Preterm Preeclampsia. N Engl J Med. 2017; 377(7): 613-622, doi: 10.1056/NEJMoa1704559, indexed in Pubmed: 28657417.

25. O'Gorman N, Wright D, Syngelaki A, et al. Competing risks model in screening for preeclampsia by maternal factors and biomarkers at 11-13 weeks gestation. Am J Obstet Gynecol. 2016; 214(1): 103.e1-103.e12, doi: 10.1016/j.ajog.2015.08.034, indexed in Pubmed: 26297382.

26. Roberge S, Villa P, Nicolaides $K$, et al. Early administration of low-dose aspirin for the prevention of preterm and term preeclampsia: a systematic review and meta-analysis. Fetal Diagn Ther. 2012; 31(3): 141-146, doi: 10.1159/000336662, indexed in Pubmed: 22441437. 